\title{
A Nonlinear Synchronization Scheme for Hindmarsh-Rose Models
}

\author{
Jung-Su Kim ${ }^{\dagger}$ and Frank Allgower*
}

\begin{abstract}
Multiple subsystems are required to behave synchronously or cooperatively in many areas. For example, synchronous behaviors are common in networks of (electro-) mechanical systems, cell biology, coupled neurons, and cooperating robots. This paper presents a feedback scheme for synchronization between Hindmarsh-Rose models which have polynomial vector fields. We show that the problem is equivalent to finding an asymptotically stabilizing control for error dynamics which is also a polynomial system. Then, an extension to a nonlinear observer-based scheme is presented, which reduces the amount of information exchange between models.
\end{abstract}

Keywords: Synchronization, Polynomial systems, Nonlinear observer and control

\section{Introduction}

Synchronization can be considered as the asymptotic coincidence of the state vectors of two (or more) systems [5]. Recently, synchronization phenomena among multiple subsystems have received much attention in various research fields including neuroscience [24], [30], [31], biology [7], [38], physics [4], [14], [17], [23], [25], [43], coordinated motion and consensus problems [16], [27], control and dynamical systems theory [5], [26], [32], and mechanical engineering [33], [35]. In these areas, it is well known that synchronous behavior among subsystems plays an important role. Cryptography based on chaotic synchronization [11] and mutual synchronization of robot manipulators [35] are representative examples in practice.

Five models have been commonly used to investigate synchronization: the Hodgkin-Huxley [21], [44], Kuramoto [1], [8], [38], Lorenz [17], [23], [41], Fitzhugh-Nagumo [24], [37], and Hindmarsh-Rose [10], [12], [13], [31] models. Note that the first two models are not in polynomial form while the rest are. For the first two models, the analysis problem of synchronization is prevalent in the literature. In other words, research is mainly directed at finding parameters leading to synchronization. For instance, focus is placed on finding a parameter called coupling strength which results in synchronization among Kuramoto models in Refs. [1], [8]. This is in contrast to the research being done on the models in polynomial form which is focused on designing a coupling function for synchronization among multiple models. For example, coupling functions are proposed for synchronization among particularly connected Fitzhugh-Nagumo models in Refs. [24] and Lorenz models in Refs. [4], [14], [17], [23]. The Hindmarsh-Rose (HR) model, one of the simplest models of oscillatory

$\dagger$ Corresponding Author: Dept. of Control and Instrumentation Engineering, Seoul National University of Technology, Korea. (jungsu@snut.ac.kr)

* Institute for Systems Theory and Automatic Control, University of Stuttgart, Germany.(allgower@ist.uni-stuttgart.de)

Received : August 6, 2009; Accepted : September 30, 2009 bursting, is particularly important in studying detailed interactions in networks containing small number of neurons. In this paper, to solve the synthesis problem for synchronization of polynomial systems, we discuss how to design a coupling function for synchronization between two HR models making use of the error dynamics, which is a first attempt of this kind.

In order to design the coupling function, we consider the error dynamics between two HR models. The coupling functions of the two models are designed so that the error dynamics are globally asymptotically stabilized. In the course of doing this, we exploit the fact that error dynamics are in the polynomial form. In other words, sum of squares (SOS) tools and semidefinite programming (SDP) ( [6], [9], [29], [34]) can be employed to design the coupling function efficiently. To implement the designed coupling function, it has to be assumed that complete state information is exchanged between models. In order to avoid this, an observer based scheme is proposed to design the coupling function for synchronization with availability of only the output information of the model. Thanks to this output feedback result, synchronization can be achieved by exchanging only the output information between models.

Several common properties can be seen in previous results. Firstly, the resulting feedback laws for synchronization are in the form of linear feedback or linear feedback with variable gain ([2], [4], [14], [17], [23], [25], [41], [43]). Secondly, feedback interaction is unidirectional ([39], [40]) in the sense that a reference model without input is employed for synchronization. Thirdly, many papers handle synchronization between two models ([17], [23], [41]). Lastly, no efficient computational tools are employed to design the feedback law.

On the contrary, the proposed scheme has many advantages compared with the previous results. Namely, the resulting feedback is nonlinear, bidirectional, and was devised using efficient computational tools: SOS and SDP. 


\section{Problem Formulation}

We first introduce the definition of synchronization under consideration.

Definition 1. Suppose that there are identical subsystems and that the $i$ th subsystem is described by

$$
\dot{x}_{i}=f\left(x_{i}, u_{i}\right), \quad i=1, \cdots, N
$$

where $x_{i} \in \mathbb{R}^{n}$ is the state of the $i$ th subsystem and $u_{i} \in \mathbb{R}^{m}$ the input (or coupling function) to the $i$ th subsystem, and all subsystems have different initial conditions. The synchronization problem is to design each coupling function so as to satisfy the two conditions below.

C1. The differences between states of the subsystems converge to zero, i.e., $x_{i}(t)-x_{j}(t) \rightarrow 0$

$(i \neq j, i, j=1, \cdots, N)$ as $t \rightarrow \infty$.

C2. The whole states are bounded

$$
\text { ( } \left.\left\|x_{i}(t)\right\|<\infty, i=1, \cdots, N\right) \text { for } t \geq 0 \text {. }
$$

Although the $N$ models are identical, the trajectories are different from each other because of the different initial conditions. In this paper, we present a feedback strategy to design each coupling function $u_{i}$ in order to solve the synchronization problem for the Hindmarsh-Rose (HR) models.

Consider the synchronization problem between the HR models. The $i$ th model is written as

$$
\begin{aligned}
\dot{x}_{i 1} & =a x_{i 2}+b x_{i 1}^{2}-c x_{i 1}^{3}-d x+I+u_{i}\left(X_{i}\right), \\
\dot{x}_{i 2} & =m-f_{i 1}^{2}-x_{i 2}+g x_{i 4}, \\
\dot{x}_{i 3} & =\mu\left[-x_{i 3}+S\left(x_{i 1}+h\right)\right], \\
\dot{x}_{i 4} & =v\left[-k x_{i 4}+r\left(x_{i 2}+l\right)\right],
\end{aligned}
$$

where $x_{i j} \in \mathbb{R}$ is the $j$ th element of the state of the $i$ th model, $u_{i} \in \mathbb{R}$ the coupling function, and all the other variables are parameters, whose values are shown in the Appendix. We denote by $X_{i}$ the stacking state of the subsystems connected to the $i$ th subsystem, i.e., $X_{i}=\left[x_{N_{i}(1)}^{T}, \cdots, x_{N_{i}\left(n_{i}\right)}^{T}\right]^{T}$ where $N_{i}$ is the set of subsystem's indices connected to the $i$ th subsystem, $N_{i}(j)$ the $j$ th element in the set $N_{i}, n_{i}$ the cardinal number of the set $N_{i}$, and $x_{i}=\left[x_{i 1}, x_{i 2}, x_{i 3}, x_{i 4}\right]^{T}$. For the notational simplicity of this paper, $\dot{x}_{i}=f\left(x_{i}\right)+G u_{i}$ is sometimes used to denote the $i$ th HR model where $f$ and $G$ are defined easily from the right hand side of Equation (1). Note that the solution of $\dot{x}_{i}=f\left(x_{i}\right)$ shows globally bounded oscillatory behavior. Therefore, the synchronization problem under consideration can be viewed as a problem of designing coupling functions for synchronization of coupled oscillators.

\section{Synchronization between Two Models}

In this section, we solve the synchronization problem for the two-model case, i.e., $N=2$. The error dynamics between two models are given as follows:

$$
\begin{aligned}
& \dot{e}_{121}=a e_{122}+b t_{12} e_{121}-c s_{12} e_{121}-d e_{123}+u_{12}\left(x_{1}, x_{2}\right), \\
& \dot{e}_{122}=-f t_{12} e_{121}-e_{122}+g e_{124}, \\
& \dot{e}_{123}=\mu\left[-e_{123}+S e_{121}\right], \\
& \dot{e}_{124}=v\left[-k e_{124}+r e_{122}\right],
\end{aligned}
$$

where $e_{i j k}=x_{i k}-x_{j k}, t_{i j}=x_{i 1}+x_{j 1}, s_{i j}=x_{i 1}^{2}+x_{i 1} x_{j 1}+x_{j 1}^{2}$, and $u_{12}\left(x_{1}, x_{2}\right)=u_{1}\left(x_{1}, x_{2}\right)-u_{2}\left(x_{2}, x_{1}\right)$. Hereafter, the arguments of the inputs $\left(X_{i}\right)$ are omitted for simplicity when it is clear from the context. In a similar manner as the system in (1), the simplified notation $\dot{e}_{12}=A\left(t_{12}, s_{12}\right) e_{12}+G u_{12}$ is used to denote the error dynamics in (2) where

$$
A\left(t_{12}, s_{12}\right)=\left[\begin{array}{cccc}
b t_{12}-c s_{12} & a & -d & 0 \\
-f t_{12} & -1 & 0 & g \\
\mu S & 0 & -\mu & 0 \\
0 & r v & 0 & -k v
\end{array}\right]
$$

and

$$
e_{12}=\left[e_{121}, e_{122}, e_{123}, e_{124}\right]^{T}
$$

If we are able to stabilize this error dynamics by designing $u_{12}$, it implies that the first condition $\mathrm{C} 1$ of the synchronization problem is fulfilled. A method of designing a stabilizing control for the error dynamics is introduced below without a proof.

Lemma 1: ([9], [10]) For the error dynamics (2), if a positive definite matrix $Q$ and polynomial matrix $M\left(t_{12}, s_{12}\right)$ exist such that the dissipation inequality below

$$
\theta^{T}\left[A\left(t_{12}, s_{12}\right) Q+G M\left(t_{12}, s_{12}\right)\right] \theta<0
$$

holds for all nonzero $\theta$, then $u_{12}=M\left(t_{12}, s_{12}\right) Q^{-1} e_{12}$ is a globally asymptotically stabilizing control for the error dynamics in (2).

This lemma is an application of a polynomial-type stabilizing control scheme ([20]) based on a Krasovskii-like Lyapunov function ([9], [10]) to the error dynamics. The fact that the dissipation inequality in (3) holds means that the derivative of a Krasovskii-like Lyapunov function is 
negative definite, which implies stability. For details of this stabilizing scheme, see [9]. Since the error dynamics is a polynomial model, the dissipation inequality (3) can be efficiently solved using SDP and SOSTOOLS ([6], [9], [24], [34]). After solving the inequality, we can obtain the following globally asymptotically stabilizing control for the error dynamics:

$$
u_{12}=\left(-0.0047+2.039 t_{12}\right) e_{122}+\left(0.996-0.006 t_{12}\right) e_{123} .
$$

Note that this feedback law is nonlinear and the value of $u_{12}$ goes to zero as $e_{12}$ does.

In principle, the stabilizing control in (4) means that, if two coupling functions $u_{1}$ and $u_{2}$ are designed such that the difference between them stabilizes the error dynamics, $\mathrm{C} 1$ is satisfied. To design such coupling functions, we revisit the definition of $u_{12}$. We recall that, if $u_{1}$ and $u_{2}$ are designed to satisfy eqnarray $\mathrm{u}$

$$
\begin{aligned}
u_{1}-u_{2}=u_{12}= & \left(-0.00472+2.039 t_{12}\right) e_{122} \\
& +\left(0.996-0.006 t_{12}\right) e_{123},
\end{aligned}
$$

Here, the coupling functions $u_{1}$ and $u_{2}$ are unknowns and $u_{12}$ is known. Therefore, we can think of Equation (5) as an underdetermined equation since there are two variables $\left(u_{1}\right.$ and $\left.u_{2}\right)$ and one equation. As a result, we can see that, if the coupling functions $u_{1}$ and $u_{2}$ are determined so that they satisfy the relation in (5) and go to zero as the error does, then $\mathrm{C} 1$ is fulfilled. To meet these two conditions, we distribute the feedback $u_{12}$ to the two coupling functions as follows: $\mathrm{u}$

$$
u_{1}=\frac{1}{2} u_{12}, u_{2}=-\frac{1}{2} u_{12} .
$$

In fact, by designing the coupling functions this way, we can be sure that the state $x_{1}$ and $x_{2}$ converge to each other. However, it is also possible for both states to diverge to infinity simultaneously. This is why the second condition $\mathrm{C} 2$ has to be fulfilled in addition.

In light of the previous coupling function design, we can observe a very important aspect of the coupling function. It seems that the error dynamics stabilization is a necessary condition for designing a coupling function. In the literature, there are many results on synchronization (or consensus). If we view those results from the viewpoint of the error dynamics, the difference between two coupling functions (e.g., that in (5)) must play the role of a stabilizer for the corresponding error dynamics if they are indeed coupling functions to achieve synchronization. This argument can be proved for linear cases in which multiple numbers of high order linear systems are connected arbitrarily [42]. The proposed coupling function for synchronization is de- signed to satisfy the inequality (3) which is a dissipation inequality for the error dynamics. This means that the coupling function makes the error dynamics dissipate its energy in order to stabilize it.

In order to investigate whether $\mathrm{C} 2$ is achieved, we recall a previous result on the synchronization of HR models [28] in which a 3rd-order HR model is considered

$$
\begin{aligned}
& \dot{x}_{1}=a x_{2}+b x_{1}^{2}-c x_{1}^{3}-d x_{3}+I+u, \\
& \dot{x}_{2}=m-f x_{1}^{2}-x_{2}, \\
& \dot{x}_{3}=\mu\left[-x_{3}+S\left(x_{1}+h\right)\right] .
\end{aligned}
$$

For this model, a passivity-like property is proved.

Lemma 2: ([28]) Consider a quadratic function

$$
W_{1}=\frac{1}{2}\left(c_{1} x_{1}^{2}+c_{2} x_{2}^{2}+c_{3} x_{3}^{2}\right),
$$

where $c_{1}, c_{2}$, and $c_{3}$ are positive. For the 3rd-order HR model, the following inequality holds

$$
\dot{W}_{1} \leq u^{T} x_{1}+H\left(x_{1}, x_{2}, x_{3}\right),
$$

This lemma says that the 3rd-order HR model has a passivity-like property, called semi-passivity. The next theorem shows that the proposed distributing method in (6) for solving the synchronization problem guarantees that $\mathrm{C} 2$ is satisfied.

Theorem 1: The state of the closed-loop system consisting of the HR model in (1) and the coupling functions determined by the rule in (6) is globally bounded.

For proof of this, see the Appendix.

This proof is based on two facts that the last equation in (1) is a globally exponentially stable system with the input $v r\left(x_{i 2}+l\right)$ and that the HR model in (1) can be viewed as an interconnected system between the system with passivity-like property in (8) and the exponentially stable last equation in (1).

Since both the conditions $\mathrm{C} 1$ and $\mathrm{C} 2$ are shown to be satisfied, the proposed method solves the synchronization problem between two HR models. Even though only a twomodel case is dealt with in this paper, if a number of models $(N \geq 3)$ is considered and they have particular interconnection (e.g., all-to-all and leader-follower structure, and if there is a spanning tree in the interconnection topology) [18],[19], the proposed method can be extended easily. Fig. 1 shows that the proposed coupling function successfully synchronized the HR models.

Remark 1: The resultant feedback is nonlinear due to the nonlinearity of the function $u_{12}$. Note that the feedback is assumed to be linear in many previous results. Nonlinear feedback is more plausible since the coupling function 
amounts to interaction between nonlinear subsystems. In the case of linear feedback, even though we can obtain similar results (e.g., error convergence rate) using the highgain approach, the resulting synchronous behavior can be sensitive to uncertainties or external disturbances.

Remark 2: The distribution method in (6) is just one example of how to fulfill $\mathrm{C} 1$ using stabilizing input (4). There can be other possibilities to distribute $u_{12}$ to $u_{1}$ and $u_{2}$. Other distribution methods are also possible as long as the resultant coupling functions $u_{1}$ and $u_{2}$ satisfy the equation in (5) and go to zero as the error $e_{i j}$ does. For example, consider the following:

$$
\begin{aligned}
u_{1}= & \left(-0.00472+2.039 x_{11}\right) e_{122} \\
& +\left(0.996-0.006 x_{11}\right) e_{123}, \\
u_{2}= & -2.039 x_{21} e_{122}+0.006 x_{21} e_{123} .
\end{aligned}
$$

Note that the undetermined equation in (5) still holds. These coupling functions are quite different from those in (6) in view of information exchange via communication between subsystems. For example, to obtain the input $u_{1}$ from (6), three pieces of information $\left(x_{21}, x_{22}, x_{23}\right)$ are required from the other subsystem. On the contrary, if the input $u_{1}$ is determined from (10), two pieces of information $\left(x_{22}, x_{23}\right)$ are needed. From the viewpoint of having the least possible information exchange between subsystems, it makes a big difference.

Remark 3: In view of the result presented above, the most important step in designing the coupling function for synchronization is to design a stabilizing input for the error dynamics. Therefore if we try to design the coupling function to solve the synchronization problem for other models, designing stabilizing input for the corresponding error dynamics becomes the crucial part of the design. In this sense, the proposed method (error dynamics stabilization and distribution) can be applicable to other polynomial-type models, because the stabilizing input for the error dynamics can be found effectively thanks to Lemma 1.

\section{Observer-based Synchronization}

In the previous section, a method to solve the synchronization problem is proposed under the assumption that all state information related to the other subsystem is available. In this section, we propose a method to establish the coupling function under the assumption that only the first state of the other subsystem, which is referred to as the output, is available. Since only the output is measurable, only a part of the state variable of the error dynamics is available while the coupling function needs to know this information. To cope with this problem, we construct a dynamic system, called an observer, to estimate the error dynamics and then we discuss how to combine the estimated error variable with the designed coupling function in the previous section. To prove the stability of the output feedback case, the following stability concept and lemma are employed.

Definition 1. ([36]) Consider the system $\dot{x}=f(x, w)$ where $x \in \mathbb{R}^{n}$ is the state and $w \in \mathbb{R}^{m}$ the external input. The system is said to be integral-input-to-state stable (iISS) if there exist a class $K_{\infty}$ function $\alpha(\cdot)$, a class $K L$ function $\beta(\cdot, \cdot)$, and a class $K$ function $\gamma(\cdot)^{1}$ such that the solution $x(t)$ of the system satisfies

$$
\alpha(\|x(t)\|) \leq \beta(\|x(0)\|, t)+\int_{s=t_{0}}^{t} \gamma(\|w(s)\|) d s
$$

where $\|\cdot\|$ denotes the standard Euclidean norm. Simply speaking, if a system is iISS, then the external input with bounded energy leads to bounded state.

Lemma 3. ([36]) If the system $\dot{x}=f(x, w)$ is iISS and the external input satisfies

$$
\int_{0}^{\infty} \gamma(\|w(s)\|) d s<\infty
$$

then the state converges to the origin.

In view of the results of the previous section, the observer-based synchronization between two subsystems boils down to designing an observer-based stabilizing output feedback control for the error dynamics defined in (2). In case the full state is measurable, it is shown that the state feedback law $u_{12}$ is a globally asymptotically stabilizing control for the error dynamics. Hereafter, it is discussed how to design output feedback for stabilization of the error dynamics using the feedback law $u_{12}$. For this purpose, a nonlinear observer is designed using a structural property of the error dynamics at first. Then, a robustness property of the feedback in (4) against the estimation error is proved to derive a nonlinear separation principle. The error dynamics in (2) can be rewritten as

$$
\begin{aligned}
& \dot{e}_{12}=A e_{12}+\phi\left(e_{12}, t_{12}, s_{12}\right)+G \hat{u}_{12} \text {, } \\
& z=\left[\begin{array}{llll}
1 & 0 & 0 & 0
\end{array}\right] e_{12}=: C e_{12} \text {, }
\end{aligned}
$$

where $z$ is the output and $A, \phi\left(e_{1}, t_{12}, s_{12}\right)$, and $\hat{u}_{12}$ are

\footnotetext{
A continuous function $\alpha:[0, a) \rightarrow[0, \infty)$ is said to belong to class $K$ if it is strictly increasing and $\alpha(0)=0$. It is said to belong to class $K_{\infty}$ if $a=\infty$ and $\alpha(r) \rightarrow \infty$ as $r \rightarrow \infty$. A continuous function $\beta:[0, c) \times[0, \infty) \rightarrow[0, \infty)$ is said to belong to class $K L$ if $\beta(a, b)$ belongs to class $K$ with respect to $a$ for a fixed $b$, and a decreasing function of $b$ for a fixed $a$ and $\beta(a, b) \rightarrow 0$ as $b \rightarrow \infty$.
} 


$$
\begin{gathered}
A=\left[\begin{array}{cccc}
0 & a & -d & 0 \\
0 & -1 & 0 & g \\
\mu S & 0 & -\mu & 0 \\
0 & v r & 0 & -v k
\end{array}\right], \\
\phi\left(e_{12}, t_{12}, s_{12}\right)=\left[\begin{array}{c}
b t_{12} e_{121}-c s_{12} e_{121} \\
-f t_{12} e_{121} \\
0 \\
0
\end{array}\right], \\
\left.\hat{u}_{12}=\left[-0.00472+2.039 t_{12}\right)\right] \hat{e}_{122} \\
+\left[0.996-0.006 t_{12}\right] \hat{e}_{123},
\end{gathered}
$$

with $(C, A)$ being observable when the parameter values in the Appendix are used, and the term $\phi\left(e_{12}, t_{12}, s_{12}\right)$ is measurable since $x_{11}$ and $x_{21}$ are. This means that all nonlinearities are measurable. It is well-known that the observer error linearization method ([15], [22]) can be applied to such a nonlinear system in order to design an observer, and that the resulting estimation error exponentially goes to zero. For the error dynamics in (12)-(13), we can design an observer as follows:

$$
\begin{gathered}
\dot{\hat{e}}_{12}=A \hat{e}_{12}+\phi\left(e_{12}, t_{12}, s_{12}\right)+G \hat{u}_{12}+L(\hat{z}-z), \\
\hat{z}=C \hat{e}_{12},
\end{gathered}
$$

where the observer gain $L$ is determined such that $A+L C$ is Hurwitz, i.e., the real parts of the eigenvalues are negative. The estimation error dynamics ${ }^{2}$ becomes

$$
\dot{e}_{o 12}=(A+L C) e_{o 12},
$$

where $e_{o 12}=\hat{e}_{12}-e_{12}$. This means that the estimation error resulting from the observer exponentially converges to the origin.

Unlike the linear system case in which the separation principle holds, designing such an observer is not the end of the design for output feedback stabilization since the system is nonlinear. In other words, the combination of the stabilizing control and the asymptotically convergent observer may not result in a stable closed-loop. To deal with this, it is required to either prove that the resulting closedloop system is robust against the estimation error or to redesign a robust control against the estimation error. A popular approach to accomplish this in the literature is to prove that the closed-loop consisting of the observer and the certainty-equivalence type control is ISS (Input-to-State Stable [3]) or iISS (integral-ISS [36]) with respect to the estimation error. Then, due to ISS or iISS of the closed-

2 In this paper, there are two kinds of error variables. Error or error dynamics denote the synchronization error $\left(e_{i j}=x_{i}-x_{j}\right)$. On the other hand, estimation error implies observer error between the error dynamics and its observer $\left(e_{o}=\hat{e}-e\right)$. loop, the exponentially stable estimation error, and the definition of the estimation error, convergence of the state to the origin is proved. The next lemma is instrumental in deriving the main result.

Lemma 2. Suppose that the unforced system $\dot{x}=f(x)$ is globally asymptotically stable. Then, the system

$$
\dot{x}=f(x)+\sigma_{1}(w)
$$

is iISS from the external disturbance $w$ to the state $x$ where $w \in \mathbb{R}^{m}$ is an external input and the function $\sigma_{1}(\cdot): \mathbb{R}^{m} \rightarrow \mathbb{R}^{n}$ satisfies $\left\|\sigma_{1}(w)\right\| \leq \sigma(\|w\|)$ with $\sigma(\cdot) \in K$.

For proof of this, see the Appendix.

The main theorem of this section is as follows.

Theorem 2. The state of the closed-loop system consisting of the error dynamics for synchronization in (2)-(3), the observer in (4)-(5), and the feedback law $\hat{u}_{12}$ converges to the origin as $t \rightarrow \infty$.

Proof: From Lemma 2, the closed-loop system

$$
\dot{\hat{e}}_{12}=A \hat{e}_{12}+\phi\left(e_{12}, t_{12}, s_{12}\right)+G \hat{u}_{12}+L C e_{o 12}
$$

is iISS with respect to $L C e_{o 12}$. In other words, we have

$$
\left\|\hat{e}_{12}(t)\right\| \leq \beta\left(\left\|\hat{e}_{12}(0)\right\|, t\right)+\int_{t_{0}}^{t} \gamma\left(\left\|L C e_{o 12}(s)\right\|\right) d s
$$

where $\beta \in K L$ and $\gamma \in K_{\infty}$. Moreover, the estimation error exponentially converges to the origin and therefore satisfies

$$
\int_{t_{0}}^{\infty}\left\|e_{o 12}(s)\right\| d s<\infty
$$

Thanks to this and Lemma 1, the observer state $\hat{e}_{12}$ goes to zero as the observer error $e_{o 12}$ does. The convergence of the error $e_{12}$ to the origin follows from the relation $e_{12}=\hat{e}_{12}-e_{o 12}$. This completes the proof.

This theorem implies that the certainty-equivalence type control $\hat{u}_{12}$ is a globally asymptotically stabilizing control for the error dynamics and goes to zero as $e_{12}$ does. Therefore, we can apply the approach in the previous section using only the first state.

\section{Summary and Outlook}

A feedback scheme to design coupling functions was proposed for synchronization of the Hindmarsh-Rose (HR) models. The coupling function was determined so that the 
error dynamics between two HR models were globally asymptotically stabilized. In view of this result, it transpired that the most important ingredient to achieve synchronization is to stabilize the error dynamics. For the HR models, the stabilizing input can be found effectively using dissipation inequality and optimization tools because of their polynomial vector fields. The proposed method was also applied to the synchronization problem of two other commonly used nonlinear models: the Lorenz and Fitzhugh-Nagumo models, which are polynomial-type models. Finally, an observer-based extension is presented where an output feedback scheme for the error dynamics was devised, and synchronization can be achieved with less information exchange. The synchronization problem for multiple models with general interconnection topology is still open as far as the nonlinear coupling function design is concerned.

\section{Acknowledgements}

This work was supported by a research fund for new faculty, SNUT 2009.

\section{Appendix}

\section{A. Proof of Theorem 1}

Lemma 2 plays a crucial role in proving the theorem. Consider the $i$ th model. We prove this theorem by showing that there exists a positive definite function $W_{i}$ such that its derivative is negative, i.e., $\dot{W}_{i}\left(x_{i}\right)<0$ outside a ball in $\mathbb{R}^{4}$. Write the 3rd-order HR model as $\dot{x}_{a}=f_{a}\left(x_{a}\right)+G_{a} u_{i}$ where $x_{a}=\left[x_{1}, x_{2}, x_{3}\right]^{T}$. Then, the 4th order HR model under consideration can be written as in the form of an interconnected system

$$
\begin{gathered}
\dot{x}_{a}=f_{a}\left(x_{a}\right)+G_{a} u_{i}+[0 g 0]^{T} x_{4}=: f_{a}\left(x_{a}\right)+G_{a} u_{i}+B x_{4}, \\
\dot{x}_{4}=-k v x_{4}+r v x_{2}+l v=: g\left(x_{4}\right)+c_{4} x_{2}+L, \quad k v>0 .
\end{gathered}
$$

Note that $\dot{x}_{4}=g\left(x_{4}\right)$ is globally exponentially stable. Consider the function as follows:

$$
W_{i}=W_{i 1}+\frac{1}{2} c_{5} x_{4}^{2}=: W_{i 1}+W_{i 2}, \quad c_{5}>0
$$

where $W_{i 1}$ denotes the Lyapunov function in (8) associated with the ith model. The derivative of this function can be written as

$$
\begin{aligned}
\dot{W}_{i}= & \frac{\partial W_{i 1}}{\partial x_{a}}\left[f_{a}\left(x_{a}\right)+G_{a} u_{i}+B x_{4}\right] \\
& +\frac{\partial W_{i 2}}{\partial x_{4}}\left[g\left(x_{4}\right)+c_{4} x_{2}+L\right] \\
= & \frac{\partial W_{i 1}}{\partial x_{a}}\left[f_{a}\left(x_{a}\right)+G_{a} u_{i}\right]+\frac{\partial W_{i 1}}{\partial x_{a}}\left[B x_{4}\right] \\
& +\frac{\partial W_{i 2}}{\partial x_{4}}\left[g\left(x_{4}\right)\right]+\frac{\partial W_{i 2}}{\partial x_{4}}\left[c_{4} x_{2}+L\right] \\
\leq & u^{T} x_{1}+H\left(x_{a}\right)+c_{2} g x_{2} x_{4}-c_{9}\left|x_{4}\right|^{2}+c_{6} c_{4} x_{4} x_{2} \\
& +c_{6} L x_{4} \\
= & c_{8} u^{2}+H\left(x_{a}\right)+c_{9}\left|x_{1}\right|^{2}+c_{8}\left|x_{2}\right|^{2}-\varepsilon\left|x_{4}\right|^{2} \\
& +c_{6} L x_{4},
\end{aligned}
$$

where all coefficient $c_{i}$ are positive and $\varepsilon>0$. The first term in (17) goes to zero as time goes by and the other terms with positive coefficients can be dominated by the term $H\left(x_{a}\right)$ which is negative outside some ball in $\mathbb{R}^{3}$. Therefore, this means that $\dot{W}_{i}\left(x_{i}\right)<0$ outside some ball in $\mathbb{R}^{4}$ and thereby showing the boundedness of the state. Applying the same argument to the other system completes the proof.

\section{B. Proof of Theorem 1}

Before proceeding further, a theorem is introduced which is instrumental in proving the lemma.

Theorem 3: The system is $\dot{x}=f(x, w)$ iISS if and only if there exists an iISS Lyapunov function $V: \mathbb{R}^{n} \rightarrow \mathbb{R}_{\geq 0}$ such that for class $K_{\infty}$ functions and $\bar{a}_{1}, \bar{a}_{2}, \gamma$ a positive definite function $\rho, V$ satisfies

$$
\bar{a}_{1}(\|x\|) \leq V(x) \leq \bar{a}_{2}(\|x\|)
$$

and

$$
\dot{V}(x(t)) \leq-\rho(\|x\|)+\gamma(\|w\|)
$$

In view of this theorem, to prove the lemma, it is sufficient to show that there exists an iISS Lyapunov function to satisfy the inequalities in (18) and (19). From the converse Lyapunov theorem, there exists a global Lyapunov function $U: \mathbb{R}^{n} \rightarrow \mathbb{R}^{+}$such that

$$
\begin{gathered}
a_{1}(\|x\|) U \leq(x) \leq a_{2}(\|x\|), \\
\dot{U}=\frac{\partial U}{\partial x} f(x) \leq-a_{3}(\|x\|), \quad\left\|\frac{\partial U}{\partial x}\right\| \leq a_{4}(\|x\|)
\end{gathered}
$$

for some class $K_{\infty}$ functions $a_{i}(i=1, \cdots, 4)$. The derivative of this Lyapunov function along $\dot{x}=f(x)+\sigma_{1}(w)$ 
has the following upper bound

$$
\begin{gathered}
\dot{U}=\frac{\partial U}{\partial x} \cdot f(x)+\frac{\partial U}{\partial x} \sigma_{1}(w) \leq-a_{3}(\|x\|)+\left\|\frac{\partial U}{\partial x}\right\|\left\|\sigma_{1}(w)\right\| \\
\leq-a_{3}(\|x\|)+a_{4}(\|x\|) \sigma(\|w\|) .
\end{gathered}
$$

As an attempt to find an iISS Lyapunov function of system (16), consider a weighted function

$$
V(r)=(\pi \circ U)(r), \quad \pi(r)=\int_{0}^{r} \frac{d s}{1+\chi(s)}
$$

and $\chi(r)=\left(a_{4} \circ a_{1}^{-1}\right)(r)$. Then, the upper bound of the derivative of the weighted function is

$$
\begin{aligned}
& \dot{V}=\frac{\frac{\partial U}{\partial x}\left(f(x) \sigma_{1}(w)\right)}{1+\chi(U(x))} \leq \frac{-a_{3}(\|x\|)}{1+\chi(U(x))}+\frac{a_{4}(\|x\|) \sigma(\|w\|)}{1+\chi(U(x))} \\
& \leq \frac{-a_{3}(\|x\|)}{1+a_{4}\left(a_{1}^{-1}\left(a_{1}(\|x\|)\right)\right)}+\frac{a_{4}(\|x\|) \sigma(\|w\|)}{1+a_{4}(\|x\|)} \\
& \leq-\rho(\|x\|)+\sigma(\|w\|) .
\end{aligned}
$$

Due to the monotone property of the function $\pi$, it is easy to see that the function $V$ is upper and lowerbounded by some class of $K_{\infty}$ functions as in (18). Therefore, the inequality in (21) implies that the function $V$ in (20) is an iISS Lyapunov function. This completes the proof.

\section{The parameters for the Hindmarsh-Rose model}

\begin{tabular}{c|c||c|c||c|c||c|c}
\hline $\mathrm{a}$ & 1 & $\mathrm{~m}$ & 1 & $\mathrm{~h}$ & 0.795 & $\mathrm{~b}$ & 3 \\
\hline $\mathrm{k}$ & 0.9573 & $\mathrm{~d}$ & 0.99 & $\mu$ & 0.001 & $\mathrm{r}$ & 3 \\
\hline $\mathrm{f}$ & 5 & $v$ & 0.0009 & $\mathrm{c}$ & 1 & $\mathrm{~g}$ & 0.028 \\
\hline $\mathrm{I}$ & 3.024 & $\mathrm{~S}$ & 4 & 1 & 1.619 & & \\
\hline
\end{tabular}

\section{References}

[1] N. Motee, A. Jadbabaie and M. Barahona, "On the stability of the Kuramoto model of coupled nonlinear oscillators," Proceedings of American Control Conference, pp.4296-4301, 2004.

[2] M. K. Ali and J.-Q. Fangnon, "Synchronization of chaos and hyperchaos using linear and nonlinear feedback functions," Physical Review E, 55(5):52855290, 1997.

[3] Murat Arcak and Petar Kokotovic, "Nonlinear observers: A circle criterion design and robustness analysis," Automatica, 37(12):1923-1930, 2001.

[4] Vladimir N. Belykh, Grigory V. Osipov, Nina Kucklanderc, Bernd Blasiusc and Jurgen Kurths, "Automatic control of phase synchronization in coupled complex oscillators," Physica D, 200:81-104, 2005.

[5] I. I. Blekhman, A. L. Fradkov, H. Nijmeijer and A. Y. Pogromsky, "On self-synchronization and controlled synchronization," Systems and Control Letters, 31:299-305, 1997.

[6] S. Boyd, L. E. Ghaoui, E. Feron and V. Balakrishnan, "Linear Matrix Inequalities in System and Control Theory, SIAM, 1994.

[7] J. Buck, "Synchronous rhythmic flashing of fireies II," Quarterly Review of Biology, 63:265, 1988.

[8] N. Chopra and M. W. Spong, "On synchronization of Kuramoto oscillators" Proceedings of IEEE Control and Decision Conference, pp.3916-3922, 2005.

[9] Christian Ebenbauer, Polynomial Control Systems: Analysis and Design via Dissipation Inequalities and Sum of Squares, PhD thesis, University of Stuttgart, Germany, 2005.

[10] Christian Ebenbauer and Frank Allgower "Analysis and design of polynomial control systems using dissipation inequalities and sum of squares," Computers and Chemical Engineering, 30:1601-1614, 2006.

[11] Rong He and P. G. Vaidya, "Implementation of chaotic cryptography with chaotic synchronization," PHYSICAL REVIEW E, 57(2):1532-1535, 1998.

[12] J. L. Hindmarsh and R. M. Rose, "A model of the nerve impulse using two first order differential equations," Nature, 296:162-164, 1982.

[13] J. L. Hindmarsh and R. M. Rose, "A model of neuronal bursting using three coupled first order differential equations," Proc. Roy. Soc. Lond. B, 221:87-102, 1984.

[14] Debin Huang, "Simple adaptive-feedback controller for identical chaos synchronization," Physical Review E, 71:37203, 2005.

[15] Alberto Isidori, Nonlinear Control Systems, 3rd Ed., Springer, 1995.

[16] A. Jadbabaie, J. Lin and A. S. Morse, "Coordination of groups of mobile autonomous agents using nearest neighbor rules," IEEE Trans. on Automatic Control, 48(6):988-1001, 2003.

[17] Junan Lu, Xiaoqun Wu, Xiuping Han, and Jinhu Lu, "Adaptive feedback synchronization of a unified chaotic system," Physics Letters A, 329:327.333, 2004.

[18] Jung-Su Kim and F. Allgower, Analysis and Design of Nonlinear Control Systems. In Honor of Alberto Isidori, chapter Nonlinear synchronization of coupled oscillators: The polynomial case, pp.361-371, Springer, 2007.

[19] Jung-Su Kim and Frank Allgower, "A nonlinear synchronization scheme for polynomial systems" In the Proceedings of American Control Conference, 2007.

[20] Min-Chan Kim, Seung-Kyu Park, Tae-Won Kim and Ho-Gyun Ahn, "A study on the design of adaptive H1 controllerpolynomial approach,” KIEE, 51(4):129-136, 2002.

[21] Seunghwan Kim, Hyungtae Kook, Sang Gui Lee and Myung-Han Park, "Synchronization and clustering in a network of three globally coupled neural oscilla- 
tions," International Journal of Bifurcation and Chaos, 8(4):731-739, 1998.

[22] Arthur J. Krener and Witold Respondek, "Nonlinear observers with linearizable error dynamics," SIAM J. Control and Optimization, 23(2):197-216, 1985.

[23] Jinhu $\mathrm{Lu}$ and Junan $\mathrm{Lu}$, "Controlling uncertain $\mathrm{Lu}$ system using linear feedback, Chaos Solutions and Fractals, 17:127-133, 2003.

[24] Deepak Mishra, Abhishek Yadav, Sudipta Ray and Prem K. Kalra, "Controlling synchronization of modified Fitzhugh-Nagumo neurons under external electrical stimulation," NeuroQuantology, 4(1):50-67, 2006.

[25] T.C. Newell, P.M. Alsing, A. Gavrielides and V. Kovanis, "Synchronization of chaos using proportional feedback," Physical Review E, 49(1):313-318, 1994.

[26] H. Nijmeijer, "A dynamical control view on synchronization," Physica D, 154:219-228, 2001.

[27] R. Olfati-Saber, "Flocking for multi-agent dynamic systems: Algorithms and theory," IEEE Trans. on Automatic Control, 51(3):401-420, 2006.

[28] Ward T. Oud and Ivan Tyukin, "Sufficient conditions for synchronization in an ensemble of Hindmarsh and Rose neurons: Passivity-based approach," In IFAC Symposium on Nonlinear Control Systems, 2004.

[29] P. A. Parrilo, Structured Semidefnite Programs and Semialgebraic Geometry Methods in Robustness and Optimization. $\mathrm{PhD}$ thesis, California Institute of Technology, 2000.

[30] F. Pasemann, "Synchronous and asynchronous chaos in coupled neuromodules," International Journal of Bifurcation and Chaos, 9:1957-1968, 1999.

[31] R. D. Pinto, P. Varona, A. R. Volkovskii, A. Szucs, Henry D. I. Abarbanel and M. I. Rabinovich, Synchronous behavior of two coupled electronic neurons," Physical Review E, 62(2):2644-2656, 2000.

[32] Alexander Pogromsky, "Observer-based robust synchronization of dynamical systems," International Journal of Bifurcation and Chaos, 8(11):2243-2254, 1998.

[33] Alexander Pogromsky and Henk Nijmeijer, "Cooperative oscillatory behavior of mutually coupled dynamical systems," IEEE Trans. on Circuit and Systems-I:Fundamental Theory and Applications, 48(2):152162, 2001

[34] S. Prajna, A. Papachristodoulou, P. Seiler and P.A. Parrilo, "SOSTOOLS and its control applications," In Positive Polynomials in Control, Lecture Notes in Control and Information Sciences, Springer, 312: 273-292, 2005.

[35] Alejandro Rodriguez-Angeles and Henk Nijmeijer, "Mutual synchronization of robots via estimated state feedback: a cooperative approach," IEEE Trans. on Control Systems Technology, 12(4):542-534, 2004.
[36] E.D. Sontag, "Comments on integral variants of ISS," Systems \& Control Letters, 34:93-100, 1998.

[37] G.-B. Stan and R. Sepulchre, "Analysis of interconnected oscillators by dissipativity theory," IEEE Trans. on Automatic Control, 52:256-270, 2007.

[38] Steven H. Strogatz, "From Kuramoto to Crawford: Exploring the onset of synchronization in populations of coupled oscillators," Physica D, 143:1-20, 2000.

[39] W. Wang and J.J.E Slotine, "On partial contraction analysis for coupled nonlinear oscillators," Biological Cybernetics, 92(1), 2005.

[40] W. Wang and J.J.E Slotine, "A theoretical study of different leader roles in networks," IEEE Trans. on Automatic Control, 51(7):1156-1161, 2006.

[41] Yanwu Wang, Zhi-Hong Guan and Hua O. Wang, "Feedback and adaptive control for the synchronization of Chen system via a single variable," Physics Letters A, 312:34-40, 2003.

[42] Peter Wieland, Jung-Su Kim, Holger Scheu and Frank Allgower, "On consensus in multi-agent systems with linear highorder agents," In Proc. 17th IFAC World Congress, pp. 1541-1546, 2008.

[43] S.S. Yang and C. K. Duan, "A mathematical theory of synchronization of self-feedback," Journal of Physics A: Mathematical and General, 30:3273-3278, 1997.

[44] Massahiko Yoshika, "Chaos synchronization in gapjunction-coupled neurons," Physical Review E, 71: 065203(R), 2005.

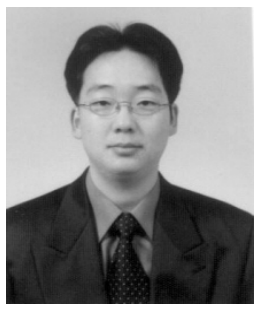

Jung-Su Kim He received his B.S., M.S., and Ph.D. degrees in electrical engineering from Korea University. He was a post-doc at CDSL, Seoul Nat'l University in 2005, at IST, University of Stuttgart, Germany, in 2006-2007, and Systems Biology Lab., University of Leicester, United Kingdom, in 2008. Since 2009 he has been with the Dept. of control and instrumentation engineering, Seoul Nat'l University of Technology, as an assistant professor. His research interest includes adaptive and predictive controls, nonlinear controls, and systems biology. (http://plaza.snut.ac.kr/ cdsl)

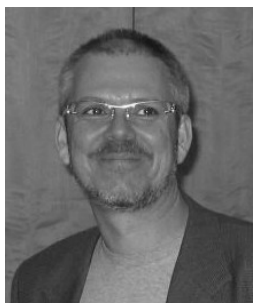

Frank Allgower Frank Allgöwer is director of the Institute for Systems Theory and Automatic Control and a professor in the Mechanical Engineering Department at the University of Stuttgart in Germany. (http://www.ist.uni-stuttgart.de/) 